UPRF-2000-18

SWAT:282

December 2000

\title{
The residual mass in Lattice Heavy Quark Effective Theory to $\alpha^{3}$ order ${ }^{1}$
}

\author{
F. Di Renzo ${ }^{a}$, and L. Scorzato ${ }^{a, b}$ \\ ${ }^{a}$ Dipartimento di Fisica, Università di Parma \\ and INFN, Gruppo Collegato di Parma, Italy \\ ${ }^{b}$ Department of Physics, \\ University of Wales, Swansea, U.K.
}

\begin{abstract}
We determine to order $\alpha^{3}$ in the quenched approximation the so-called residual mass in the lattice regularisation of the Heavy Quark Effective Theory. We follow a gauge-invariant strategy which exploits the fact that this mass term dominates the exponential decrease of perturbative Wilson loops at large perimeters. Our computational tool is Numerical Stochastic Perturbation Theory. The new coefficient we compute is crucial to improve the determination of the $(\overline{\mathrm{MS}})$ mass of the $b$-quark from lattice simulations of the Heavy Quark Effective Theory.
\end{abstract}

\footnotetext{
${ }^{1}$ Research supported in part by Italian MURST under contract 9902C68583, by I.N.F.N. under i.s. PR11 and by EU Grant EBR-FMRX-CT97-0122.
} 


\section{Introduction}

Heavy Quark Effective Theory (HQET) is proving to be a particularly useful tool for phenomenological studies in heavy flavour physics (see [1] for a review and references therein to original papers). In this approach physical quantities are expanded as series in inverse powers of the heavy quark masses $m_{Q}>>\Lambda_{Q C D}$. The Lagrangian density of the effective theory may be written as

$$
\mathcal{L}_{H Q E T}=\bar{h} D_{4} h
$$

where $D_{4}$ is the (gauge) covariant time derivative; $h$ is the bi-spinor describing the only degrees of freedom of the heavy quark that are relevant in this approximation. Soon after its introduction it was realized that while HQET could appear conceptually simple, there was a variety of theoretical subtleties one should face in order to profit from its application. Not surprisingly, many of these subtleties have to do with the definition of the heavy quark mass $m_{Q}$ itself. First of all it was realized [2] that the theory must be invariant by a redefinition of $m_{Q}$ which leaves the momentum $P_{Q}^{\mu}$ fixed:

$$
P_{Q}^{\mu}=m_{Q} v^{\mu}+k^{\mu} .
$$

In fact the mass of a quark is not a well defined physical quantity, and the separation of $P_{Q}^{\mu}$ into a large and a small $\left(k^{\mu} \sim \Lambda_{Q C D}\right)$ component is to some extent arbitrary. The requirement of invariance by redefinition of $m_{Q}$ leads to the introduction of a "covariant" derivative in terms of which the HQET Lagrangian can acquire a mass term for the static quark. It was noted however that even so the expansion in $1 / m_{Q}$ is not well defined and other ambiguities show up. In dimensional regularisation they appear as UV renormalons in the matrix elements of HQET and IR renormalons in the coefficient functions that match to QCD [3]. In the lattice regularisation problems show up as non-perturbative power divergences in the inverse lattice spacing [4]. The ambiguities in the coefficient functions are expected to cancel with those in the HQET matrix elements. In order to perform such a subtraction and to obtain a properly defined expansion the expansion parameter itself $\left(m_{Q}\right)$, first of all, must be defined in a way free of ambiguities. The pole mass $m_{\text {pole }}$ proved not to be a good definition [3]. The authors of [5] introduced a lattice non-perturbative definition for the quark mass (the so-called subtracted pole mass) which is free of renormalon ambiguities and power divergences

$$
m_{Q}^{S} \equiv m_{H}-\bar{\Lambda}
$$

In the previous formula $m_{H}$ is the mass of an hadron containing the heavy quark, and $\bar{\Lambda}$ is the non-perturbative definition of a subtracted binding energy

$$
\bar{\Lambda} \equiv \mathcal{E}-\delta \bar{m}
$$

$\mathcal{E}$ is the "naive" binding energy, computed as the decay constant of the correlation function of two axial currents $\left(A_{4}=\overline{h(x)} \gamma_{4} \gamma_{5} q(x), q(x)\right.$ being a light quark) evaluated with the action in Eq. (1):

$$
\begin{aligned}
C(t) & =\sum_{\vec{x}}\left\langle A_{4}(\vec{x}, t) A_{4}(\overrightarrow{0}, 0)^{\dagger}\right\rangle \\
& \sim Z^{2} \exp (-\mathcal{E} t)
\end{aligned}
$$


The other term in Eq. (4) is a non-perturbative definition of the so-called residual mass counterterm. The latter is just the quantity we are concerned with in this paper. It is a linearly divergent mass counterterm which quantum corrections generate in the lattice regularisation, contrary to dimensional regularisation. In the non perturbative approach we have just referred to, it is fixed by the large time behaviour of the quark propagator $S_{h}^{i j}(x, y)$ ( $i, j$ colour indexes):

$$
\delta \bar{m} \equiv-\lim _{t \rightarrow \infty} \frac{1}{a} \log \left(\frac{\left|S_{h}^{i i}(\vec{x}, t+a ; 0,0)\right|}{\left|S_{h}^{i i}(\vec{x}, t ; 0,0)\right|}\right) .
$$

Both $m_{Q}^{S}$ and $\bar{\Lambda}$ are non perturbative, well defined quantities and may be evaluated by numerical simulations on the lattice [6, 7]. This is not at all a trivial observation. Both $\mathcal{E}$ and $\delta \bar{m}$ are linearly divergent quantities on their own and only the result of the subtraction in Eq. (4) is a well defined, divergences free quantity, which also makes well defined Eq. (3). However, the subtracted pole mass is still a long distance quantity and in order to be able to extract physical predictions from lattice HQET it is necessary to match the definition of $m_{Q}^{S}$ to another mass definition such as the mass $\overline{m_{Q}}$ of $\overline{\mathrm{MS}}$. In fact the coefficient functions of the $1 / m_{Q}$ expansion are typically computed in perturbation theory in dimensional regularisation [5]. The relation between $m_{Q}^{S}$ and the $\overline{\mathrm{MS}}$ mass of the $b$-quark $\overline{m_{b}}\left(\overline{m_{b}}\right)$ has been computed in [6, 7] and exploited to compute the mass of the $b$-quark. The strategy goes as follows. The pole mass (we write $m_{b}^{\text {pole }}$ having in mind the pole mass for the $b$-quark) can be related to the $\overline{\mathrm{MS}}$ mass in perturbation theory

$$
\overline{m_{b}}\left(\overline{m_{b}}\right)=m_{b}^{p o l e}\left[1+\sum_{n=0}^{\infty}\left(\frac{\alpha_{\mathbf{s}}\left(\overline{m_{b}}\right)}{\pi}\right)^{n+1} D_{n}\right]
$$

Note that in this relation coefficients $D_{n}$ with $n \leq 2$ are known [8, 9]. By matching the propagator in QCD to its lattice HQET counterpart (we closely follow [7]) one can then relate the pole mass to the binding energy and the physical mass of an hadron, for example the B meson mass $\left(M_{B}\right)$. This relation is just the perturbative version of Eq. (3)

$$
m_{b}^{\text {pole }}=M_{B}-\mathcal{E}+\sum_{n=0}^{\infty} \alpha_{\mathrm{s}}^{n+1} \frac{X_{n}}{a}+\mathcal{O}\left(1 / m_{b}\right) \equiv M_{B}-\mathcal{E}+\delta m+\mathcal{O}\left(1 / m_{b}\right)
$$

This time the residual mass term $(\delta m)$ has naturally emerged from the perturbative computation of the heavy quark propagator on the lattice. We slightly change notation to stress that $\delta m$ is the perturbative residual mass. By substitution one gets

$$
\overline{m_{b}}\left(\overline{m_{b}}\right)=\left[M_{B}-\mathcal{E}+\sum_{n=0}^{\infty}\left(\alpha_{\mathbf{s}}\left(\overline{m_{b}}\right)\right)^{n+1} \frac{X_{n}}{a}\right]\left[1+\sum_{n=0}^{\infty}\left(\frac{\alpha_{\mathbf{s}}\left(\overline{m_{b}}\right)}{\pi}\right)^{n+1} D_{n}\right]
$$

Note that the last expression asks for both a non-perturbative $(\mathcal{E})$ and a perturbative $(\delta m)$ computation. $\delta m$ is still in charge of cancelling the linear divergence of $\mathcal{E}$, even if now the cancellation is in perturbation theory, so that it is incomplete. What one also gets from Eq. (10) is a delicate cancellation of renormalon ambiguities as well: the renormalon in the expansion of $\delta m$ cancels the one in the perturbative relation between the pole and $\overline{\mathrm{MS}}$ masses and this is the key issue. This cancellation asks first of all for the use of the same coupling in both perturbative expansions. It also asks for high orders in the perturbative expansions. Since, as we have already said, $D_{2}$ is known, this means that $X_{2}$ has to be computed. In the end it turns out [7] that the ignorance of this term is the main source of error in the 
final determination of $\overline{m_{b}}\left(\overline{m_{b}}\right)$. This observation ends this introductory review which sets the motivations for our computation: the purpose of this paper is to compute $\delta m$ to $\alpha_{\mathbf{s}}^{3}$ order $\left(X_{2}\right)$ in the quenched approximation. Our computational tool is Numerical Stochastic Perturbation Theory (NSPT) [10]. We point out that while $X_{0}$ has been known for a long time, $X_{1}$ has only recently been computed [11], while the only results available for $X_{2}$ are at the moment the ones presented at the Lattice 99 conference [12, 13], which we will compare our result to. We stress that the present computation is still a partial result, since we stay within the quenched approximation. We emphasize that an unquenched, non-perturbative computation of the binding energy is already available, so that an extension of our result to include the fermionic loops contribution is compelling. We are actually working on it. With this respect the present computation is a first step which nevertheless we think is useful for at least a couple of reasons. First of all, we will show that NSPT can get the result to an accuracy which is good enough for the purpose at hand. On top of that we will discuss how our result has an impact on the final accuracy on $\overline{m_{b}}\left(\overline{m_{b}}\right)$, at least in the quenched approximation. In the next section we briefly summarize the strategy of our computation and discuss the numerical analysis which needs to be performed. In section 3. we report our results and their impact. Finally in section 4 . we state our conclusions.

\section{The computational strategy}

An obvious choice in order to compute the residual mass would be the computation of the heavy quark propagator. This would ask for gauge fixing. While our computational tool (NSPT) can manage such a task, it turns out that gauge invariant computations are in NSPT both easier and less time demanding (for further technical details on this and other points concerning the method itself, see [10]). The fact that a gauge invariant computation can be set up in order to obtain the residual mass has already been pointed out and exploited in [11] and also in [12, [13]. In order to understand, consider a gauge invariant loop such as a Wilson $(W)$ or a Polyakov $(P)$ loop. There are three sources of divergences for such a quantity [14]. A first one is the logarithmic divergence that can be as usual absorbed in the definition of the renormalised coupling. An additional logarithmic divergence appears if the contour has an angle (i.e. such a problem is there in the case of a Wilson loop and not for a Wilson line or a Polyakov loop); this is usually referred to as the corner divergence. A third, linear divergence is the one we are interested in, resulting in (consider for example a Wilson loop and note that the divergence exponentiates and shows up as a factor)

$$
\langle W\rangle=\exp (-c L / a) W_{l o g}
$$

In the above formula $L$ is the length of the loop and $a$ the cutoff scale at small distance (we have of course already in mind the lattice spacing). The notation $W_{\log }$ reminds that only logarithmic divergences are left. The Wilson line (also called P-line) one would need to compute in order to obtain the lattice heavy quark propagator would of course show the same divergences. It is interesting to note that HQET was not there in the early eighties when people first got interested in the so-called "phase factors" in Gauge Theories. Still of course the physical picture was already there, the linearly divergent factor being regarded as mass renormalisation of a test particle if one treats the loop as an effective amplitude for a test particle moving along the trajectory given by the loop itself. Martinelli and Sachrajda [11] 
exploited the above considerations in order to compute $\delta m$ to second order by extracting the linearly divergent contributions from the whole set of integrals contributing to the Feynmann graphs for Wilson loops $W(R, T)$ of generic size $R \times T$ in lattice perturbation theory. The latter ones had already been computed to order $\alpha_{\mathrm{s}}^{2}$ in [15]. In [12] our group pinned down a first number for $\delta m$ to third order (basically its order of magnitude) by performing a fit of squared Wilson loops of different sizes $L$ to the expression given in Eq. (11). At the same Lattice 99 conference the Fermilab group presented a value for the same quantity by considering a Polyakov loop $P$ in which the mass term contribution was isolated by the Coulomb (self-)interaction of the heavy quark [13]. Let us now introduce the method we exploited in this work. We think that a very neat way to extract $\delta m$ goes along the lines we now proceed to describe. Consider the quantity

$$
V(R) \equiv \lim _{T \rightarrow \infty} V_{T}(R) ; \quad V_{T}(R) \equiv \log \left(\frac{W(R, T-1)}{W(R, T)}\right) .
$$

$W(R, T)$ are Wilson loops of size $R \times T$. Note the dependence on $T$ which is removed by taking the $T \rightarrow \infty$ limit; we do not further comment on this point at the moment (there will be a lot to say about that later). Eq. (12) goes of course back to the old subject of Creutz's ratios defined to extract the static inter-quark potential. It is for example considered in [15]. It actually achieves the separation of the different (divergent) contributions we are interested in. First of all, note that the corner divergences are cancelled between numerator and denominator, the number of corners being the same. We now compute everything in lattice perturbation theory. In the end one is left with

$$
V(R)=2 \delta m+V_{\text {Coul }}(R) .
$$

In the above expression one reads the sum of $2 \delta m$ and of the actual Coulomb static interquark potential $V_{\text {Coul }}(R)$. The logarithmic divergence connected to the renormalisation of the charge is contained in the potential. The latter actually defines a renormalised coupling in the so-called potential scheme according to

$$
V_{\text {Coul }}(R) \equiv-C_{F} \frac{\alpha_{\mathrm{V}}(R)}{R} .
$$

Note that till now we have made no use of the fact that we are going to compute all the quantities in the lattice scheme, apart from having explicitly written the linear divergence as $\delta m$. By computing everything in lattice perturbation theory and by separating the linear from the logarithmic divergence one actually computes the coupling in the potential scheme $\left(\alpha_{\mathrm{V}}\right)$ as an expansion in the lattice coupling $\left(\alpha_{0}\right)$, i.e. one computes the matching between the couplings in the potential and the lattice scheme. In general at any given order such a matching is known once one knows the ratios between the $\Lambda$ parameters and the differences between non-universal coefficients of the $\beta$-functions $\left\{b_{i}, i \geq 2\right\}$ in the two schemes. If we stop at the order we are interested in

$$
\alpha_{2}(s \mu)=\alpha_{1}(\mu)+C_{1}(s) \alpha_{1}(\mu)^{2}+C_{2}(s) \alpha_{1}(\mu)^{3}+\ldots
$$

where

$$
\begin{aligned}
& C_{1}(s)=-2 b_{0} \log s+2 b_{0} \log \frac{\Lambda_{2}}{\Lambda_{1}} \\
& C_{2}(s)=C_{1}(s)^{2}-2 b_{1} \log s+2 b_{1} \log \frac{\Lambda_{2}}{\Lambda_{1}}+\frac{b_{2}^{(2)}-b_{2}^{(1)}}{b_{0}}
\end{aligned}
$$


Now the point is that at this order the matchings of both the potential [16] and the lattice [17] schemes to the $\overline{\mathrm{MS}}$ scheme are known. Since the $\overline{\mathrm{MS}} \beta$-function coefficients are known to three loops, one obtains from the above matchings both the ratios of $\Lambda$ parameters and the $b_{2}$ coefficients for both the potential and the lattice schemes. This in turns means that the matching between the couplings in the potential and the lattice scheme is known to the order we need. This defines our strategy. From what we have just said it follows that in Eq. (13) everything but $\delta m$ is known, i.e.

$$
V(R)=2 \delta m-\frac{C_{F}}{R}\left(\alpha_{0}+c_{1}(R) \alpha_{0}^{2}+c_{2}(R) \alpha_{0}^{3}+\ldots\right)
$$

where

$$
\begin{aligned}
& c_{1}(R)=2 b_{0} \log R+2 b_{0} \log \frac{\Lambda_{V}}{\Lambda_{0}}, \quad \log \frac{\Lambda_{V}}{\Lambda_{0}}=4.4076 \\
& c_{2}(R)=c_{1}(R)^{2}+2 b_{1} \log R+2 b_{1} \log \frac{\Lambda_{V}}{\Lambda_{0}}+\frac{b_{2}^{(V)}-b_{2}^{(0)}}{b_{0}}, \quad \frac{b_{2}^{(V)}-b_{2}^{(0)}}{b_{0}}=8.5794
\end{aligned}
$$

and

$$
\delta m=\sum_{n \geq 0} \overline{X_{n}} \alpha_{0}^{n+1}
$$

the expression for $\alpha_{\vee}$ having been taken from Eq. (15). The "perfect" strategy would then be as follows. Compute $V(R)$ in lattice perturbation theory and compare order by order the result to Eq. (16): the coefficients in the expansion of $\delta m$ are the only unknown quantities, that could be easily obtained. In practice we had to go through a number of approximations: we will discuss how they have to be taken under control and their impact on the final result estimated as a final error. First of all, we worked not in infinite volume, but on a lattice of fixed $32^{4}$ volume, as it is obviously requested by our computational tool (NSPT). We will further comment on this point later. Of course, this implies that we do not have access to the real $V(R)$, but only to its (finite volume) $V_{T}(R)$ approximations. Of course for small $R$ and $T$ and for finite lattice spacing the expression in Eq. (16) is distorted by lattice artifacts. On top of all the above considerations one should also keep in mind that the results of NSPT are affected by statistical errors and their impact should be carefully assessed as well. In the end, what we did was to fit the $V_{T}(R)$ to the asymptotic expression for $V(R)$. The approximations $V_{T}(R)$ were in turn obtained from the computation of $W(R, T)$ for a wide range of $R$ and $T$. A first, obvious point to make is that taking lattice artifacts under control asks for having both $R$ and $T / R$ fairly large. The choice of a $32^{4}$ lattice was motivated by the compromise between these observations and the computing requirements of NSPT in terms of both memory and flops. Having said that, it is also important to point out from the very beginning that there are a couple of attitudes one can take with respect to the fits we had to perform. First, one can take the coefficients in the expansion of $\delta m$ as the only unknown parameters, taking for all the other parameters entering Eq. (16) their asymptotic, lattice artifacts free values. On the other side, one can study the stability of results by fitting the other constants in Eq. (16) as well. This can give an idea of the extent to which our computations are good approximations of the infinite volume, lattice artifacts free results.

\section{Results}




\section{$3.1 \delta m$ to third order}

We computed $W(R, T)$ on a $32^{4}$ lattice for all $R, T \leq 16$. Since we needed to compute $\delta m$ to order $\alpha_{0}^{3}$, these expansions had to be computed to the same order. We refer people interested in these results themselves to a future review publication 10 in which they will be made available. In that context a detailed discussion of the statistical errors associated to NSPT results will be presented. For the purposes at hand here we only need to observe that a percent precision [/ of order $\leq 10^{-3}$ is easy to obtain in the range of $R$ and $T$ we were interested in. To do better than this would have been relatively easy, which thing we decided not to do in this exploratory study in the quenched approximation. Of course the statistical error is magnified by the extraction of the potential, since the log series are there. In the end, a percent precision only of order $10^{-2}$ was obtained for the potential at order $\alpha_{0}^{3}$, again in the range of $R$ and $T$ we took into account. As far as the choice of the latter is concerned, we have already said that reducing lattice artifacts and keeping finite size effects under control were the major issues. Note that while the overall volume was kept fixed, both the $R / T$ and $R$ scales were relevant. It is trivial to observe that there is no finite size scaling that could be set up within these constraints. What we did was to constrain the values for $R$ and $T$ this way: for every choice of $T$ in the range $12 \leq T \leq 15$ the fits were done within an interval of $3 \div 4$ values of $R$ subjected to the constraints that $a$ ) the central value should be such that $R / T$ stayed around a value $R / T \sim 3$ and b) $R \geq 3$. It turns out that with such a procedure one is in a good position to perform the fit in which every parameter but the coefficients in the expansion of $\delta m$ is taken as given by the asymptotic expressions. The results so obtained were regarded as approximations of the infinite volume, lattice artifacts free results. Of course on top of the statistical errors a systematic error has to be taken into account connected to the choice of the fitting interval, i.e. of $R$ and $T$. Note that as approximations of the infinite volume results, the $V_{T}(R)$ should be loosely dependent on $T$. These can be tested also by studying the stability of results with respect to averaging as in $\left(V_{T}(R)+V_{T+1}(R)\right) / 2$. While this gives no new information at first and second order, it turns out that it lets you gain something at $\alpha_{0}^{3}$ order, where the impact of statistical errors (which are bigger at this order) is a bit relieved by this averaging procedure. In order to gain some insight into the effectiveness of the procedure we have just described (that is, the one in which only $\delta m$ is to be fitted), we first of all tried to reproduce the coefficients that were already known in the expansion of $\delta m$. Expressing them in an expansion in the bare lattice coupling $\alpha_{0}$, these coefficients are $\overline{X_{0}}=2.1173, \overline{X_{1}}=11.1520$. We got

$$
\overline{X_{0}}=2.118(2) \quad \overline{X_{1}}=11.14(3) .
$$

$\mathrm{T}$ The agreement with the analytical, infinite volume results is surprisingly good. The errors are in practice dominated by the systematic uncertainty connected to the choice of the fitting interval. Such an uncertainty was estimated to be given by the standard deviation pertaining to the sample of results of fitting procedures within the constraints we described a few lines above. For an extra peace of mind these systematic errors and the statistical ones were added not in quadrature. Before making further comments, one can proceed straight to repeat the same procedure at order $\alpha_{0}^{3}$, which gives the result we are interested in as

$$
\overline{X_{2}}=86.2(6)
$$

\footnotetext{
${ }^{2}$ We quote the worst precision, that is the one on order $\alpha_{0}^{3}$.
} 

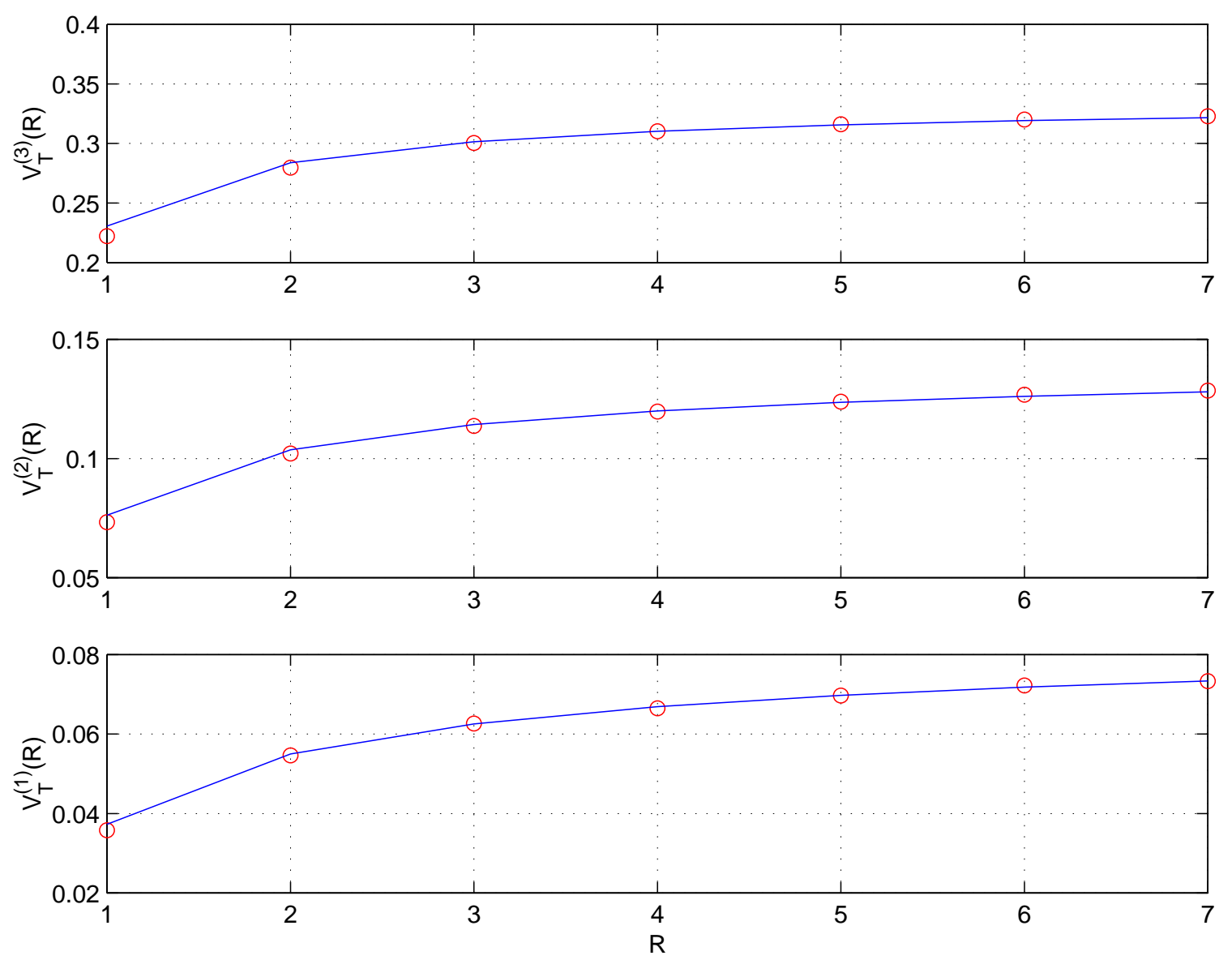

Figure 1: A typical result of the fitting procedure described in the text. The three plots refer to first, second and third order of the potential. The solid lines are the appropriate orders as given by Eq. (16) once $\delta m$ has been fitted, while circles are the approximations $V_{T}(R)$. In this case $T=14$.

This time an appreciable contribution is also coming from statistical errors in the computation of the loops and of the potential, even if the systematic uncertainty connected to the choice of the fitting interval nevertheless stays as the major one. Again, these different errors were added not in quadrature. In the figure we plot an example of the results one gets by this procedure: by fitting values for the coefficients in the expansion of $\delta m$ one obtains that order by order the approximation of $V(R)$ falls on the curve given by Eq. (16). One should keep in mind that the effectiveness of the procedure is actually magnified by the figure. This in particular true for the highest order, for which the fitted result is a huge shift with respect to the final order of magnitude of plotted data. The sensitivity with respect to such a huge shift can be easily underestimated if one only looks at the figure. Note by the way that the huge shift gives a good idea of the impact of the renormalon on the expansion. Now the question comes whether the error bars obtained in the first procedure we described are to be fully trusted. As we have already pointed out the effectiveness in reproducing the known coefficients within fairly tiny errors is impressive. As it is obvious, this is nevertheless only known a posteriori and in this sense it is not a proof, but only a hint at the reliability of the method. As stressed, our first procedure relied on the assumption that the $V_{T}(R)$ were good 
approximations of the infinite volume, lattice artifacts free $V(R)$, the only sources of errors being the statistical ones and the systematic effects of the choice of the fitting intervals. As we have already suggested, a second attitude can be taken with respect to fitting the $V_{T}(R)$ to the expression given by Eq. (16). This does not assume the other coefficients entering the formulas as given by their asymptotic, lattice artifacts free values. This means at leading order to fit also the coefficient $C_{F}$, at second order also the ratio of the $\Lambda$ parameters and at third order the difference in the $\beta$-function coefficients $b_{2}$ as well. This is an indirect test of how asymptotic our approximations are. One should anyway always keep in mind that this is not a good starting point for a proper finite size analysis, since in all our procedure there is a finite fixed volume and a couple of scales around. We will anyway assume the typical variation of coefficients with respect to this procedure as a sensible bound on finite size and lattice artifacts effects. The constraints we imposed before on the choice of the fitting intervals had to be somehow released in order to be able to fit more than one parameter. Anyway, no more than two parameters were fitted simultaneously (that is, at every order the relevant coefficient in the expansion of $\delta m$ plus another constant). This was obtained by letting every order inherit fixed values for other parameters. For example: the second order fit was in charge of fitting $\overline{X_{1}}$ and the ratio of $\Lambda$ parameters, inheriting the value for $C_{F}$ either from first order fit or from the infinite volume result (of course also variations with respect to these variants were taken into account). Depending again on the choice of fitting intervals, we found a rough bound of $10 \div 15 \%$ on the variations of all the parame-

ters entering the Coulomb potential, including $\frac{b_{2}^{(V)}-b_{2}^{(0)}}{b_{0}}$. Ironically, the latter used to be for us a first source of confidence in our results. Given our ignorance of reference [16], at the beginning we fitted this coefficient together with the third order $\delta m$, assuming for the other parameters their asymptotic values. The reasonable agreement of the result of the fit with [16] turned out to be a reasonable hint at the reliability of our result for $\overline{X_{2}}$. As far as the values of the coefficients of the expansion of $\delta m$ we found instead a rough bound of $1 \%$. Note how this bound appears surprisingly huge with respect to the deviation of our results from the exact ones for first and second order. As we have already observed, this is something which can be anyway verified a posteriori. A possible step forward in the estimate of finite size effects could be to repeat the whole procedure on a different volume, which thing we propose to do in the future unquenched study. At the moment, taking into account also the last considerations, for an extra piece of mind we assume as our final result

$$
\overline{X_{2}}=86.2(0.6)(1.0)
$$

Our result is quite apart from the one [13] presented at the Lattice 99 conference by the Fermilab group, suggesting a fairly big impact of systematics. We point out that the computational tools are as a matter of fact quite different. What we plan to do in the future (for the unquenched computation) is to follow also their approach based on Polyakov instead of Wilson loops, which thing allows a fine finite size analysis.

\subsection{The impact of the result}

It is now the proper time to address the question of the impact of our result. As a matter of fact the result presented in this paper has already been known for some time to people involved in the field. Lubicz's review on quark masses at the Lattice 2000 conference [18] has 
already referred to the impact of the knowledge of $X_{2}$ in the determination of the $b$-quark mass, at least in the quenched approximation. Basically, things go as follows 19 (this is what one would refer to as a Next to Next to Next to Leading Order - NNNLO - quenched calculation of the $b$-quark mass). The lines along which the computation can go have already been widely presented in the Introduction, the final formula being Eq. (10). In order to understand what one achieves with the result for $X_{2}$ a good point is the comparison with the lower order (NNLO) determination'?. Of course all the following considerations about the impact of NNNLO hold for the quenched case, given the limitation of our result. The residual error due to unknown perturbative corrections in Eq. (10) is halved with respect to NNLO and the new order improves the convergence of the series. Given that the determination of the quark mass can be got from results obtained at various values of $\beta$, a remarkable point is that the dependence of the final result for the quark mass on the lattice spacing is smaller than in NNLO and very weak. In the end, it turns out that the overall error on the mass is of order $1 \div 2 \%$, the contributions being equally split between perturbative and non-perturbative ones (this final error was at NNLO dominated by the ignorance of $X_{2}$ ).

\section{Conclusions}

We computed the perturbative expansion of the residual mass term in lattice Heavy Quark Effective Theory to order $\alpha_{0}^{3}$. This is an important building block in a renormalon safe determination of the $b$-quark mass from lattice simulations. Errors appear to be under a fairly good control and the impact of the result on the (unquenched) determination of the quark mass is quite important. Nevertheless, the result we presented is still a partial one: going for unquenching is compelling. The latter is our proposed next step forward. We will do that trying to still improve on systematics.

\section{Acknowledgments}

We thank G. Burgio and M. Pepe for having collaborated with us at an early stage of a work which eventually also merged in this paper. We are extremely grateful to V. Gimenez and V. Lubicz for illuminating discussions on the subject and to G. Martinelli and C.T. Sachrajda not only for what we learned from them on the subject, but also for their constant interest and encouragement. We also thank P. Mackenzie and the Fermilab group for having shared with us their understanding of the subject. F. D.R. acknowledges support from both Italian MURST under contract 9902C68583 and from I.N.F.N. under i.s. PR11. L. S. acknowledges support from EU Grant EBR-FMRX-CT97-0122.

\section{References}

[1] M. Neubert, Physics Reports 245, 259 (1994)

\footnotetext{
${ }^{3}$ The reader can get all the details for lower orders results in references [10, 11, 7.
} 
[2] A. F. Falk, M. Neubert, M. E. Luke, Nucl. Phys. B 388, 363 (1992).

[3] M. Beneke, V.M. Braun, Nucl. Phys. B 426, 301 (1994).

[4] L. Maiani, G. Martinelli and C. T. Sachrajda, Nucl. Phys. B 368, 281 (1992).

[5] G. Martinelli and C. T. Sachrajda, Phys. Lett. B 354, 423 (1995).

[6] M. Crisafulli, V. Giménez, G. Martinelli and C. T. Sachrajda, Nucl. Phys. B 457, 594 (1995).

[7] V. Giménez, L. Giusti, G. Martinelli and F. Rapuano, Journ. of High Energy Physics 03, 18 (2000).

[8] K.G. Chetyrkin and M. Steinhauser, Phys. Rev. Lett. 83, 4001 (1999); K.G. Chetyrkin and A. Retey, Nucl. Phys. B 583, 3 (2000).

[9] K. Melnikov and T. van Ritbergen, Phys. Lett. B 482, 99 (2000).

[10] F. Di Renzo, G. Marchesini, P. Marenzoni and E. Onofri, Nucl. Phys. B 426, 675 (1994). For a recent update see also F. Di Renzo, L. Scorzato, Nucl. Phys. B (Proc. Suppl.) 83, 822 (2000) and F. Di Renzo and L. Scorzato, "Understanding Numerical Stochastic Perturbation Theory: a status report for Lattice Gauge Theories", in preparation.

[11] G. Martinelli and C. T. Sachrajda, Nucl. Phys. B 559, 429 (1999).

[12] G. Burgio, F. Di Renzo, M. Pepe, L. Scorzato, Nucl. Phys. B (Proc. Suppl.) 83, 935 (2000).

[13] G.P. Lepage, P.B. Mackenzie, N.H. Shakespeare, H.D. Trottier, Nucl. Phys. B (Proc. Suppl.) 83, 866 (2000).

[14] V. S. Dotsenko, S. N. Vergeles. Nucl. Phys. B 169, 527 (1980)

[15] U. Heller, F. Karsh, Nucl. Phys. B 251, 254 (1985).

[16] Y. Schröeder, Phys. Lett. B 447, 321 (1999).

[17] M. Luescher, P. Weisz, Nucl. Phys. B 452, 234 (1995). B. Alles, A. Feo, H. Panagopoulos, Nucl. Phys. B 491, 498 (1997).

[18] V. Lubicz, hep-lat/0012003, to be published in the Proceedings of the Lattice 2000 conference. See also S. Collins, hep-lat/0009040, to be published in the Proceedings of the Confinement IV conference.

[19] V. Giménez and G. Martinelli, private communication. 